\title{
Diffraction-limited CCD imaging with faint reference stars
}

\author{
R. N. Tubbs ${ }^{1}$, J. E. Baldwin ${ }^{2}$, C. D. Mackay $^{1}$, and G. C. Cox ${ }^{3}$ \\ 1 Institute of Astronomy, Madingley Road, Cambridge CB3 0HA, UK \\ 2 Cavendish Astrophysics Group, Cavendish Laboratory, Madingley Road, Cambridge CB3 0HE, UK \\ e-mail: jeb@mrao.cam.ac.uk \\ 3 Nordic Optical Telescope, Apartado 474, E-38700 Santa Cruz de La Palma, Canarias, Spain \\ e-mail: cox@not.iac.es
}

Received 4 March 2002 / Accepted 26 March 2002

\begin{abstract}
By selecting short exposure images taken using a CCD with negligible readout noise we obtained essentially diffraction-limited $810 \mathrm{~nm}$ images of faint objects using nearby reference stars brighter than $I=16$ at a $2.56 \mathrm{~m}$ telescope. The $F W H M$ of the isoplanatic patch for the technique is found to be 50 arcseconds, providing $\sim 20 \%$ sky coverage around suitable reference stars.
\end{abstract}

Key words. techniques: high angular resolution - instrumentation: detectors - globular clusters: individual: M 13

\section{Introduction}

In recent observations with the Nordic Optical Telescope (NOT) we demonstrated that selection of the best images from a large dataset of short exposures can provide essentially diffraction-limited images at wavelengths shorter than $1 \mu \mathrm{m}$ from well-figured ground-based telescopes as large as $2.5 \mathrm{~m}$ diameter (Baldwin et al. 2001). Images of bright stars derived from the best $1 \%$ of exposures of $5 \mathrm{~ms}$ duration under good seeing had Strehl ratios as high as 0.3 at $810 \mathrm{~nm}$. The use of the brightest speckle for image selection, shifting and co-adding provides a significant improvement in resolution over recentring and selection techniques based upon the image centroid (see Nieto \& Thouvenot 1991 and references therein).

The applicability of this technique to faint astronomical targets depends on the probability that a sufficiently bright reference star lies within the isoplanatic patch prevailing at the times of the selected exposures. This probability is expected to be very much higher for selected exposures than for adaptive optics (AO) at the same wavelength for the following reasons: the atmospheric phase variations at the times of the selected exposures are about $1 \mathrm{rad}$ rms over the whole mirror aperture $\left(\sim 6-7 r_{0}\right)$ rather than $\sim r_{0}$ for non-conjugate $\mathrm{AO}$, so the isoplanatic patch is expected to be correspondingly large; light from the whole aperture $\left(\sim 6-7 r_{0}\right)$ contributes to the short exposure images rather than $\sim r_{0}$ for $\mathrm{AO}$ wavefront sensors; and since selection of exposures is a passive activity, exposures can be at least 10 times longer than those necessary for

Send offprint requests to: R. N. Tubbs,

e-mail: rnt20@ast.cam.ac.uk servo-correction in $\mathrm{AO}$, further increasing the limiting magnitude.

If the telescope mirror itself contains phase errors, then the best exposures will be those where the atmosphere has partially corrected these errors. The atmospheric phase variations may then be larger than $1 \mathrm{rad}$ rms over the whole aperture, giving less of an improvement in isoplanatic patch size over AO. A similar situation may arise if a good exposure occurs when phase variations cancel out between two independent atmospheric layers.

A separate consideration is whether the read-out noise of the detector compromises the quality of the final image obtained by summing the selected short exposures. The development of CCDs with negligible readout noise (Mackay et al. 2001) has eliminated this noise penalty for fast frame rate imaging. The use of such CCDs for observations involving image selection should provide yet further improvement in the limiting magnitude of the reference star which can be used and also the limiting sensitivity to faint target objects in the field around the reference star.

The observations described here were designed to test the performance of the exposure selection technique at low light levels using a low noise CCD and to investigate the size of the isoplanatic patch for the technique. We were also interested in determining the quality of relative photometry and astrometry within globular cluster images using a range of reference star magnitudes.

\section{Observations and data reduction}

On the nights of 2001 July 5 and 6 we undertook high frame-rate imaging observations at the Cassegrain focus of 
the NOT using a camera built around a low-noise Marconi CCD. The camera comprised a front-illuminated $576 \times 288$ frame-transfer CCD65 with $20 \times 30 \mu \mathrm{m}$ pixels cooled in a liquid nitrogen dewar to $120 \mathrm{~K}$ to minimise dark current. The CCD was run by an AstroCam 4100 controller modified to provide a variable voltage clock signal for the output gain register of the CCD. We used frame rates between $18 \mathrm{~Hz}$ and $140 \mathrm{~Hz}$, with sub-array readout where necessary. The CCD temperature was raised slightly on the second night (July 6) so as to improve the charge transfer efficiency. Short exposure images taken with high output register gain showed time-varying pattern noise with an amplitude equivalent to $\sim 0.5$ detected photons.

The $\mathrm{f} / 11$ beam at the focus was converted to $\mathrm{f} / 60$ using a single achromat, giving an image scale of $27 \times$ 40 milliarcsec per pixel and a total imaging area of $11.5 \times$ 15.4 arcsec. In order to investigate the image quality achieved at large angular separations from the reference star, the camera optics were designed so that light from two regions of the sky separated by 25 arcsec could be superimposed on the CCD. This allowed science targets to be imaged at separations of up to 30 arcsec from the reference star. All the observations were made at $810 \mathrm{~nm}$ with a top-hat filter of $120 \mathrm{~nm}$ bandwidth and with no autoguider in operation.

Good exposures were selected on the basis of Strehl ratios calculated from each short exposure image of the reference star, a technique demonstrated previously in Baldwin et al. (2001). The spurious bright spots found in images taken with our CCD65 detector (Mackay et al. 2001) did not occur frequently enough to have a significant effect on the reconstructed images, although a small number of frames with bright spots close to the reference star were excluded from our analyses.

The accuracy of measurement of the Strehl ratio and position of the brightest speckle was improved by convolving the individual frames with the diffraction-limited telescope point-spread function before these parameters were calculated. The peak intensity in the convolved images represents the location where correlation with the diffraction-limited point-spread function is maximised, providing a very good estimate for the location and intensity of a diffraction-limited speckle within the original image. After frames have been selected based on the Strehl ratio for the brightest speckle found, the corresponding unprocessed exposures are sinc resampled, shifted and coadded to produce the final image.

\section{Results}

The size of the isoplanatic patch in Strehl-selected exposures was deduced from observations of the binary stars HD 203991, 8 Lac and 61 Cygnii, which have separations of $0.6,22$ and 30 arcsec respectively. These were all bright enough that the selection and image shift correction for exposures was free of the effects of small number statistics in photon detection. One component of each binary was used as the reference to select the best $1 \%$ of the exposures, for

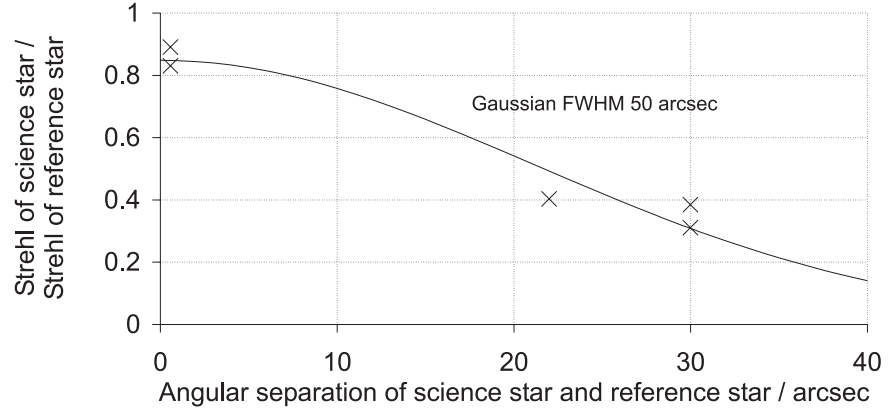

Fig. 1. The Strehl ratio of each science object divided by the Strehl ratio for the corresponding reference star is plotted against the angular separation between science object and reference star.

which both the image full width at half-maximum intensity $(F W H M)$ and Strehl ratio of the other component was also measured. Figure 1 shows the fractional reduction in Strehl ratio of the second component as a function of angular separation from the reference star. A Gaussian fit to the points gives 50 arcsec for the $F W H M$ of the isoplanatic patch for these selected frames, taken during a period in which the mean seeing was 0.51 arcsec. The image FWHM for objects 30 arcsec from the reference star using the best $1 \%$ from a run of 4000 exposures of 61 Cygnii was 130 milliarcsec, only slightly poorer than the FWHM of $\sim 80$ milliarcsec expected for a well sampled diffractionlimited point-spread function. The measured $F W H M$ is increased to 230 milliarcsec when selecting the best $10 \%$ of exposures, and 300 milliarcsec when selecting all the exposures for a shift-and-add image.

The effect of reference star magnitude on the quality of the resulting image was studied using observations with the full field of view of the CCD in a densely populated region of $\mathrm{M}$ 13. The $3.4 \mathrm{MHz}$ pixel rate of the CCD controller limited the frame rate for these observations to $18 \mathrm{~Hz}$, allowing image motion to blur the exposures. This limited the Strehl ratios for reconstructed images to $\leq 0.16$, with image $F W H M$ of $\sim 100$ milliarcsec. Data was taken from 6 runs of 1000 frames each. The selection of the best $1 \%$ of the 6000 exposures worked successfully with reference stars as faint as $I=15.9$. The effects of "over-resolution" on faint reference stars (Nieto \& Thouvenot 1991) were avoided by using other objects in the field for image quality measurements. Figure 2 shows the variation in the Strehl ratio and $F W H M$ of nearby stars when a range of different stars are used as the reference for image selection, shifting and adding. $I$ band stellar magnitudes were taken from Cohen et al. (1997). The Strehl ratios with reference stars of $I=13.8$ and $I=15.9$ are 0.13 and 0.065 respectively, a substantial improvement over $\sim 0.019$ for conventional astronomical images generated from each of the individual runs. Figure 3 shows a comparison of the image quality in these cases. The elongation in Fig. 3c may be due to image drift over the $55 \mathrm{~s}$ unguided exposure.

Given a suitable reference star, what combination of exposures gives the best image? The simple method 
a)
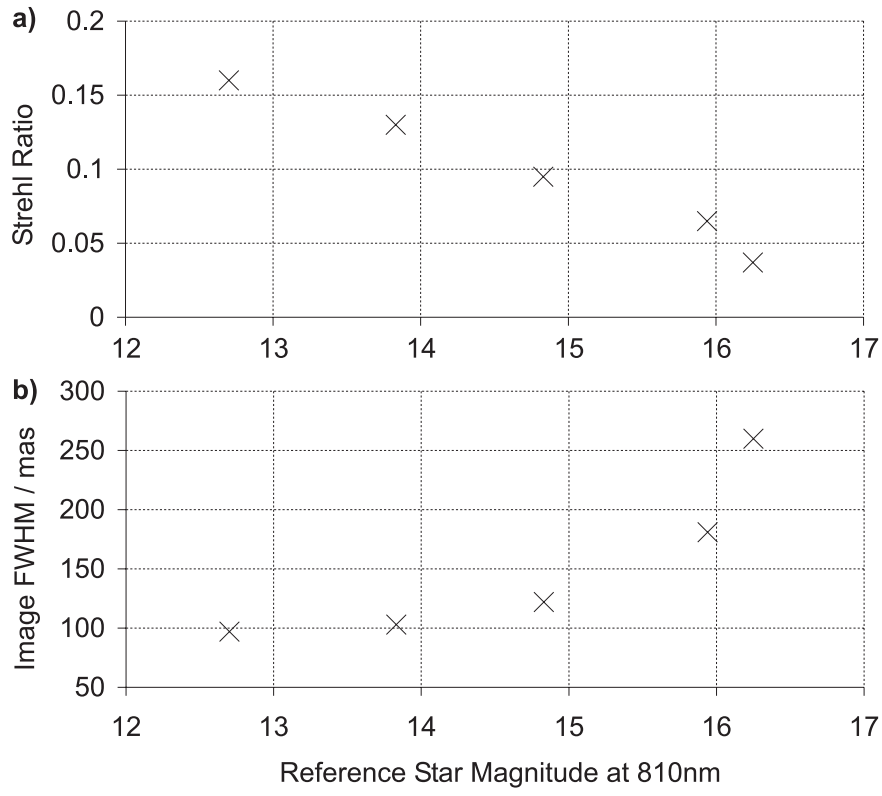

Fig. 2. a, b) Image resolution for a range of different reference stars in M 13. a) Typical Strehl ratios for stars within a few arcsec of the reference star, for a range of reference star magnitudes. b) Typical image $F W H M$ in milliarcsec for these stars.

a)

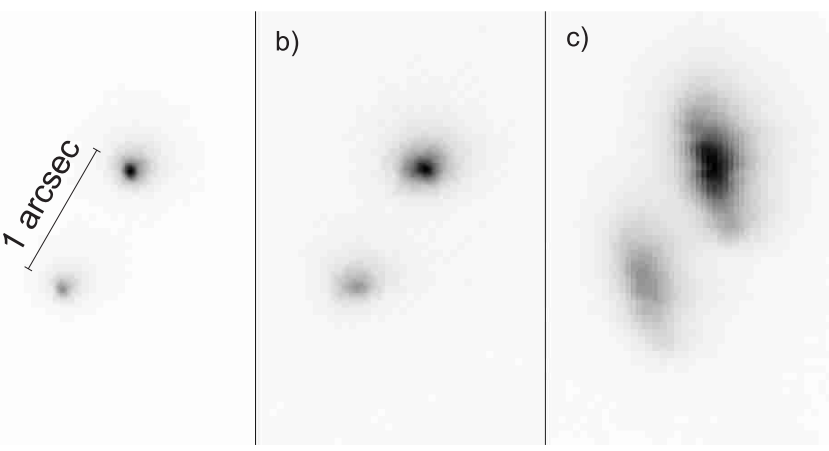

Fig. 3. $\mathbf{a}-\mathbf{c}$ ) Image resolution in $M 13$ with different reference stars. a) Image selection, shifting and adding using an $I=13.8$ reference star 2.5 arcsec away. Image $F W H M=$ 100 milliarcsec, Strehl ratio $=0.13$. b) Image selection, shifting and adding using an $I=15.9$ reference star $2.9 \operatorname{arcsec}$ away. Image $F W H M=180$ milliarcsec, Strehl ratio $=0.065$. c) Conventional image of M 13 produced by summing together 1000 of the raw frames without shifting to compensate for image motion. Image $F W H M=570 \times 390$ milliarcsec, Strehl ratio $=0.019$.

applied so far is to select the best $x \%, x$ being chosen to balance good Strehl ratio against adequate sensitivity. If the noise is Gaussian, a better solution would be to weight each exposure proportionally to the square of the signal-to-noise ratio (snr) for the quantity of interest. For example, detection of a faint stellar image with Strehl ratio $S$ against a uniform noise background would require weighting $\propto S^{2}$. Since the value of $S$ for a faint target is unmeasurable in a single exposure, how well does the
Strehl ratio for the reference star, $S_{\text {ref }}$, represent that for the target?

To check this, data on the binary stars $\zeta$ Boötis (from May 2000; Baldwin et al. 2001), 61 Cygnii and a close pair of stars in M13 were reanalysed. The exposures in each run were ordered by $S_{\text {ref }}$, placed in 100 equal bins starting with the best $1 \%$, then the next $1 \%$ and so on. The exposures in each bin were shifted and co-added and the resulting Strehl ratio for the companion star was plotted against $S_{\text {ref }}$ (Figs. $4 \mathrm{a}-\mathrm{c}$ ). All three plots show a linear relation between the Strehl ratios. The scatter of the points about the line increases from (a) to (c) mainly because of the decreasing number of exposures in each bin, but also in (c) due to photon statistics for the faint reference star. The latter also biases $S_{\text {ref }}$, offsetting the mean line from the origin. The interesting conclusion is that $S_{\text {ref }}$ is a good measure of $S$ for the target objects, even for comparatively low Strehl ratios; there seems no lower limit at which the correlation collapses. In practice, the limiting sensitivity using exposures weighted by $S_{\text {ref }}^{2}$ was determined by pattern noise produced by the camera electronics. Exposure selection was found to give better rejection of this pattern noise, and has been used for the remainder of our analyses.

Figure 5a shows a high resolution image of a field near the core of M 13 generated by selecting the $10 \%$ of exposures with the highest Strehl ratios from 6000 taken at $18 \mathrm{~Hz}$ frame rate. The $I=12.7$ reference star used for exposure selection and recentring has been labelled. Typical stellar FWHM in this image are $\sim 120$ millarcsec. Slight asymmetry in the faint halos around stars may relate to reduced charge transfer efficiency on the CCD at low signal level. Cross-sections through the stars with magnitudes 13.8 and 14.9 labelled $X$ and $Y$ are shown in Fig. 5b.

In order to asses the image quality, the 60 best exposures of M 13 were separated into two groups of 30 . The exposures in each group were shifted and added together giving two independent images of the field in M 13. These images were then convolved with the diffraction-limited telescope point-spread function. Measurements were made of the location and intensity of the brightest pixel for each star, providing crude relative astrometry and photometry within the images. The rms difference in astrometry between the two independent datasets was found to be 6 milliarcsec, and the rms difference in stellar magnitudes was 0.02 . The limiting magnitude for a $5 \sigma$ detection of a star above the noise in these images was $I \simeq 19$.

\section{Conclusions}

The observations described here show that: (i) the selection of short exposure images can provide high resolution imaging up to 30 arcsec from a reference star; (ii) Strehl ratios and image shift can be accurately calculated with reference stars as faint as $I=15.9$; and (iii) assuming a mean density of 400 stars per square degree at the South Galactic Pole with $I \leq 15.9$ (Reid \& Gilmore 1982), $8 \%$ of the South Galactic Pole region is within range of a suitable 

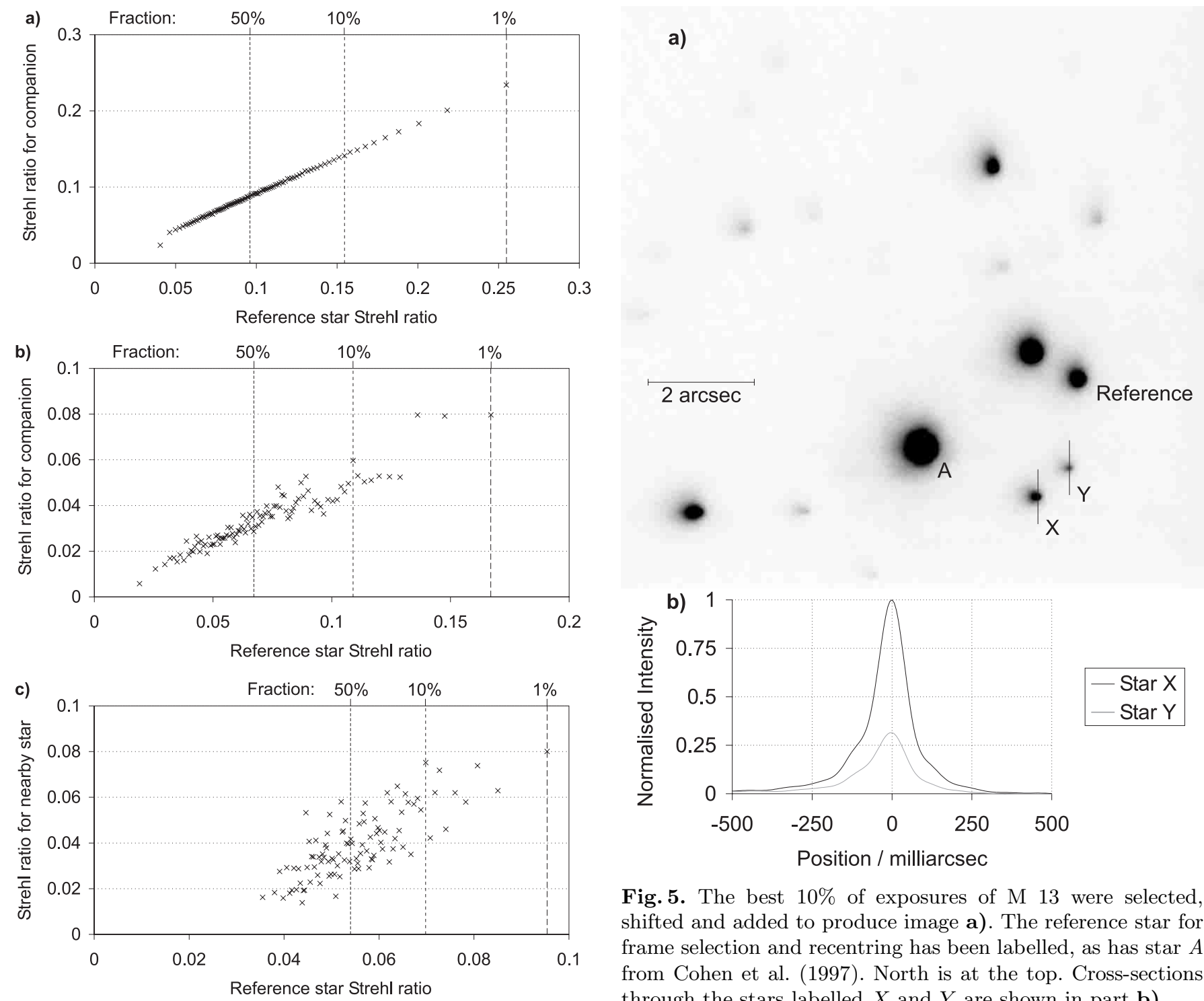

Fig. 5. The best $10 \%$ of exposures of M 13 were selected, shifted and added to produce image a). The reference star for frame selection and recentring has been labelled, as has star $A$ from Cohen et al. (1997). North is at the top. Cross-sections through the stars labelled $X$ and $Y$ are shown in part b).

Fig. 4. a-c) Strehl ratios for reference star and field star in shift-and-add images using selected short exposures. a) Exposures of $\zeta$ Boötis were sorted according to the Strehl ratio measured on the $\mathrm{A}$ component (reference star) into 100 groups of 232 exposures. The exposures in each group were shifted and co-added. Figure 4a shows the resulting Strehl ratio of the binary companion plotted against the reference star Strehl ratio. The vertical lines indicate the reference Strehls given by the best, the 10th best and the 50th best group of exposures. b) This process repeated for 4000 exposures taken on the 30 arcsec binary star 61 Cygnii. c) 1000 exposures from M 13 were analysed in the same way. The reference star had a magnitude of $I=15.9$. Strehl ratios for a nearby $I=12.7$ star are shown.

reference star. If the mean stellar density over the whole sky is 3.3 times higher, then $\sim 20 \%$ of the sky will be within range of suitable reference stars with our current detector. The recent development of thinned low-noise CCDs and improvements to our camera electronics should improve the limiting reference star magnitude, allowing high resolution imaging over $\geq 25 \%$ of the sky. The faintest

point source detected at a $5 \sigma$ level from $10 \%$ of exposures selected in an hour of observing would be $I \geq 21$ with our old camera or $I \sim 23$ with the improved instrument.

Acknowledgements. The Nordic Optical Telescope is operated on the island of La Palma jointly by Denmark, Finland, Iceland, Norway and Sweden, in the Spanish Observatorio del Roque de los Muchachos of the Instituto de Astrofisica de Canarias.

\section{References}

Baldwin, J. E., Tubbs, R. N., Cox, G. C., et al. 2001, A\&A, 368, L1

Cohen, R. L., Guhathakurta, P., Yanny, B., Schneider, D. P., \& Bahcall, J. N. 1997, AJ, 113, 669

Mackay, C. D., Tubbs, R. N., Bell, R., et al. 2001, Proc. SPIE, 4306, 289

Nieto, J.-L., \& Thouvenot, E. 1991, A\&A, 241, 663

Reid, N., \& Gilmore, G. 1982, MNRAS, 201, 73 